\title{
Gene expression profiling of KBH-A42, a novel histone deacetylase inhibitor, in human leukemia and bladder cancer cell lines
}

\author{
MOO RIM KANG $^{1 *}$, JONG SOON KANG $^{1 *}$, JEONG WOOK YANG $^{1}$, BO GEUN KIM ${ }^{1}$, \\ JIN-AH KIM ${ }^{1}$, YEONG NANG JO ${ }^{1}$, KIHO LEE ${ }^{1}$, CHANG WOO LEE ${ }^{1}$, KI HOON LEE $^{1}$, JIEUN YUN ${ }^{1}$, \\ HWAN MOOK KIM ${ }^{1}$, GYOONHEE HAN ${ }^{2}$, JONG SEONG KANG ${ }^{3}$ and SONG-KYU PARK ${ }^{4}$ \\ ${ }^{1}$ Bioevaluation Center, Korea Research Institute of Bioscience and Biotechnology, Cheongwon, Chungbuk 363-883; \\ ${ }^{2}$ Department of Biotechnology, Yonsei University, Seoul 120-749; ${ }^{3}$ College of Pharmacy, Chungnam National University, \\ Daejeon 305-764; ${ }^{4}$ College of Pharmacy, Korea University, Yeongi, Chungnam 339-700, Republic of Korea
}

Received April 27, 2011; Accepted August 26, 2011

DOI: $10.3892 / \mathrm{ol} .2011 .430$

\begin{abstract}
The aim of this study was to investigate the anti-tumor activity of $\mathrm{KBH}-\mathrm{A} 42$, a novel synthetic histone deacetylase (HDAC) inhibitor. KBH-A42 was shown to significantly suppress the proliferation of all 14 human cancer cell lines tested. Among these cell lines, the human leukemia cell line K562 was the most sensitive, whereas the UM-UC-3 bladder cancer cells were the least sensitive. Additionally, in a human tumor xenograft model using Balb/c nude mice, $\mathrm{KBH}-\mathrm{A} 42$ was shown to significantly inhibit the growth of K562 tumors, although it only slightly inhibited the growth of UM-UC-3 tumors. The results of flow cytometry analysis and caspase 3/7 activation assays showed that the growth inhibition of K562 cells by KBH-A42 was mediated, at least in part, by the induction of apoptosis, but its growth inhibitory effects on UM-UC-3 cells were not mediated by apoptotic induction. In an effort to gain insight into the mechanism by which KBH-A42 inhibits the growth of cancer cells, a microarray analysis was conducted. Four genes were selected from the genes that were down-regulated or up-regulated by KBH-A42 and confirmed via reverse transcription-polymerase chain reaction as follows: Harakiri $(H R K)$, tumor necrosis factor receptor superfamily, member $10 \mathrm{~b}$ (TNFRSF10B), PYD and CARD domain containing protein gene (PYCARD) and tumor necrosis factor receptor superfamily, member 8 (TNFRSF8). Collectively, the in vitro and in vivo results suggested that KBH-A42 exhibits anti-cancer activity, but various types of cells may be regulated differentially by KBH-A42.
\end{abstract}

Correspondence to: Dr Song-Kyu Park, College of Pharmacy, Korea University, Chochiwon, Yeongi, Chungnam 339-700, Republic of Korea

E-mail: spark123@korea.ac.kr

*Contributed equally

Key words: histone deacetylase inhibitor, cell cycle arrest, apoptosis, microarray

\section{Introduction}

The anti-cancer potential of histone deacetylase (HDAC) inhibitors has been broadly acknowledged $(1,2)$. These compounds block HDAC activity, resulting in profound increases in the acetylation state of the chromatin, which in turn affects chromatin structure and the regulation of gene expression (3). Previous studies using cDNA arrays showed that the expression of as many as $7-10 \%$ of genes were altered in cell lines of leukemia, multiple myeloma, and carcinomas of the colon, bladder, kidney, prostate and breast, when cultured for up to $48 \mathrm{~h}$ with butyrate, TSA, MS-275, vorinostat or FK228 (depsipeptide), using a 2-fold change as the cut-off value (4-8). The patterns of alterations of gene expression are similar for different HDAC inhibitors, but definite differences were clearly induced by different agents in various transformed cell types (9). The development of HDAC inhibitor resistance is a major concern, as is the case with any novel anti-tumor therapy. In preclinical studies, resistance to HDAC inhibitorinduced transformed cell death was noted in human bladder carcinoma cells (T24) and prostate cancer cells (PC3) $(10,11)$. However, the mechanisms underlying HDAC inhibitor resistance remain to be clarified.

In this study, we evaluated the functional effects of KBH-A42 on the growth of various cancer cell types $(12,13)$. Additionally, K562 (leukemia) cell lines were the most sensitive to KBH-A42, and UM-UC-3 (bladder cancer) cells were the least sensitive. Furthermore, in a human tumor xenograft model using Balb/c nude mice, KBH-A42 was shown to significantly inhibit the growth of K562 tumors, but slightly inhibited the growth of UM-UC-3 tumors. In an effort to determine the reason for the differential response of K562 and UM-UC-3 cells to KBH-A42, cDNA microarray analyses were conducted and confirmed by reverse transcription-polymerase chain reaction (RT-PCR) on the two cell types.

\section{Materials and methods}

Chemicals, cell lines and animals. All reagents were purchased from Sigma-Aldrich (St. Louis, MO, USA) unless stated otherwise. KBH-A42 was synthesized and supplied 
by Dr Gyoonhee Han at Yonsei University (Seoul, Republic of Korea). KBH-A42 was dissolved in dimethyl sulfoxide (DMSO) and freshly diluted in culture media for all in vitro experiments. Female BALB/c-nu mice were purchased from SLC (Hamahatsu, Japan) and maintained as previously described (14). All animals were permitted to acclimate to the local environment for at least 1 week prior to use. The cell lines CaSki, HeLa, Hep 3B, SNU709, A549, AsPC-1, PC-3, A375, LOX-IMVI, M14 and AZ521 were cultured in RPMI-1640 (Gibco BRL, Carlsbad, CA, USA); the UM-UC-3, K562 and KB-3-1 cell lines were cultured in Dulbecco's modified Eagle's medium (DMEM; Gibco BRL). All media were supplemented with $10 \%$ fetal bovine serum (Hyclone, Logan, UT, USA), $2 \mathrm{mM}$ L-glutamine, $100 \mathrm{U} / \mathrm{ml}$ of penicillin and $100 \mu \mathrm{g} / \mathrm{ml}$ of streptomycin. The cells were maintained at $37^{\circ} \mathrm{C}$ in $5 \% \mathrm{CO}_{2}$ humidified air.

Cell proliferation assay. Cells were plated at $0.8-1.5 \times 10^{4}$ cells/ well in 96-well plates, incubated overnight and treated with $\mathrm{KBH}-\mathrm{A} 42$ for $48 \mathrm{~h}$. Cell proliferation assays were performed using a cell proliferation kit II (XTT Roche Applied Science Mannheim, Penzberg, Upper Bavaria, Germany) in accordance with the manufacturer's instructions. The XTT labeling mixture was prepared by mixing 50 volumes of $1 \mathrm{mg} / \mathrm{ml}$ sodium 3'-[1-(phenylaminocarbonyl)-3,4-tetrazolium]-bis (4methoxy-6-nitro) benzenesulfonic acid hydrate with 1 volume of $0.383 \mathrm{mg} / \mathrm{ml}$ of $\mathrm{N}$-methyldibenzopyrazine methyl sulfate. This XTT labeling mixture was subsequently added to the cultures and incubated for $2 \mathrm{~h}$ at $37^{\circ} \mathrm{C}$. Absorbance was measured at $490 \mathrm{~nm}$, with $650 \mathrm{~nm}$ as a reference wavelength.

Human tumor xenografts in nude mice. The $\mathrm{K} 562$ and UM-UC-3 cells were injected subcutaneously into female BALB/c-nu mice. When the tumor volume reached $100 \mathrm{~mm}^{3}$, the mice were distributed randomly and treated with vehicle, KBH-A42 (100 mg/kg body weight, i.p., QD), or doxorubicin (2 $\mathrm{mg} / \mathrm{kg}$ body weight, i.p., Q2D) for 14 days. Following 14 days of treatment, the mice were sacrificed and all of the tumors were removed and weighed.

Apoptosis analysis. Apoptosis was analyzed using an annexin V-FITC apoptosis detection kit II (BD Biosciences, NJ, USA) in accordance with the manufacturer's instructions. In brief, the cells were plated at $2-4 \times 10^{6}$ cells/dish in $100-\mathrm{mm}$ dishes, incubated overnight and treated for $24 \mathrm{~h}$ with the indicated concentrations of KBH-A42. The cells were harvested, washed in phosphate-buffered saline (PBS) and combined with a binding buffer containing annexin V-FITC and propidium iodide (PI). Following $15 \mathrm{~min}$ of incubation in the dark, the cells were analyzed via flow cytometry using a FACSCalibur flow cytometer (BD Bioscience).

Caspase 3/7 assay. The activities of caspases 3 and 7 were determined using a Caspase-Glo 3/7 ${ }^{\mathrm{TM}}$ assay (Promega, Madison, WI, USA) in accordance with the manufacturer's instructions. In brief, the cells were plated at $0.8-1.5 \times 10^{4}$ cells/well in 96-well plates, incubated overnight and then treated for $24 \mathrm{~h}$ with the indicated concentrations of KBH-A42. The culture supernatants were transferred to a turbid microtiter plate and mixed with equal volumes of Proluminescent
Table I. Effect of KBH-A42 and SAHA on the proliferation of various cell lines.

\begin{tabular}{llcc}
\hline \multirow{2}{*}{ Cell line } & \multirow{2}{*}{ Origin } & \multicolumn{2}{c}{$\mathrm{GI}_{50}(\mu \mathrm{M})$} \\
\cline { 3 - 4 } & & KBH-A42 & ADR \\
\hline UM-UC-3 & Bladder & 1.41 & 0.68 \\
K562 & Blood & 2.12 & 0.19 \\
CaSki & Cervix & 2.48 & 0.19 \\
HeLa & Cervix & 3.54 & 0.77 \\
KB-3-1 & Cervix & 4.81 & 0.51 \\
Hep 3B & Liver & 7.55 & 1.03 \\
SNU709 & Liver & 7.71 & 0.47 \\
A549 & Lung & 7.08 & 0.16 \\
AsPC-1 & Pancreas & 9.88 & 1.50 \\
PC-3 & Prostate & 4.44 & 0.76 \\
A375 & Skin & 3.65 & 0.11 \\
LOX-IMVI & Skin & 3.78 & 0.62 \\
M14 & Skin & 7.49 & 0.21 \\
AZ521 & Stomach & & 0.21 \\
\hline
\end{tabular}

Cells were plated at $9 \times 10^{3}$ cells/well in 96-well plates, incubated overnight and treated for $48 \mathrm{~h}$ with various concentrations of KBH-A42. Cell proliferation was evaluated with an XTT assay, as described in Materials and methods. GI50 values were determined via non-linear regression analysis using GraphPad Prism version 4.0 software.

caspase 3/7 substrate (Promega). Following $1 \mathrm{~h}$ of incubation at $37^{\circ} \mathrm{C}$, luminescence was measured with a VICTOR ${ }^{\mathrm{TM}}$ light (PerkinElmer, NJ, USA).

RNA extraction. In brief, cells were plated at $2-4 \times 10^{6}$ cells/ dish in $100-\mathrm{mm}$ dishes, incubated overnight and treated for $24 \mathrm{~h}$ with KBH-A42 at the indicated concentrations. RNA was isolated with a Qiagen RNeasy Plus Mini kit according to the manufacturer's instructions, and its quantity and purity were evaluated using the A260/280 ratio (SmartSpec ${ }^{\text {TM }} 3000$, Bio-Rad, CA, USA).

cDNA microarray and data analysis. Profiling of gene expression was analyzed using a Gene Agilent human 44K 4-plex chip (Digital Genomics, Korea) in accordance with the manufacturer's instructions. In brief, approximately $2 \mu \mathrm{g}$ of human total RNA was reverse-transcribed with Cy3- or Cy5-conjugated dUTP (Amersham Pharmacia Biotech, Uppsala, Sweden), respectively. After labeling the reaction for $1 \mathrm{~h}$ at $42^{\circ} \mathrm{C}$, the $\mathrm{Cy} 3$ - and Cy5-labeled cDNA probes were mixed together and hybridized to a microarray slide for $16 \mathrm{~h}$ at $60^{\circ} \mathrm{C}$. The microarray was scanned with a GenePix $4000 \mathrm{~B}$ scanner (Axon Instruments, CA, USA), and the scanned image was analyzed with GenePix v6.0 software to determine the gene expression ratio. Raw data were normalized via the locally weighted scatter-plot smoother (LOWESS) normalization method. Normalized spot intensities were calculated into gene expression ratios between the control and treatment groups. 
A

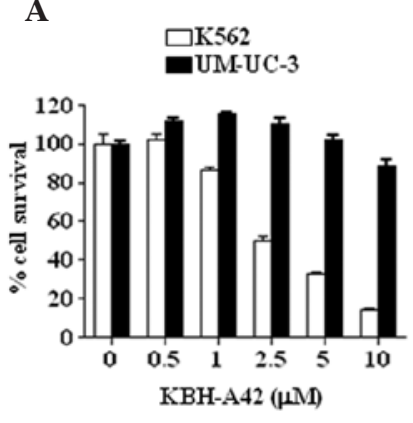

C

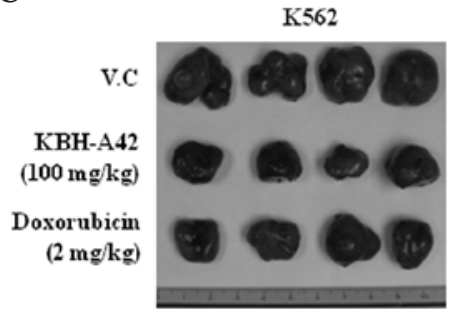

B

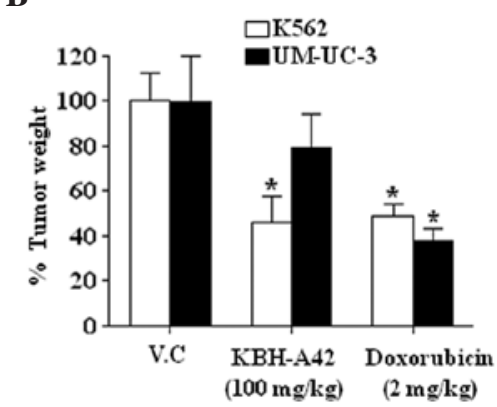

UM-UC-3

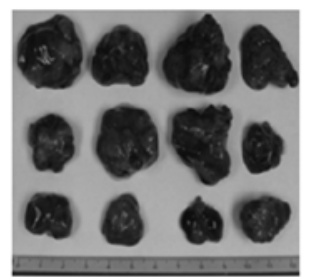

Figure 1. Effect of KBH-A42 on the growth of K562 and UM-UC-3 cells in vitro and in vivo. (A) Cells were treated with the indicated concentrations of KBH-A42 for $24 \mathrm{~h}$. Cell proliferation was determined with a XTT assay. (B) BALB/c-nu mice were injected s.c. with K562 or UM-UC-3 cells. When the tumor volumes reached $100 \mathrm{~mm}^{3}$, the mice were treated with vehicle, KBH-A42 (100 mg/kg, QD) or ADR (2 mg/kg, Q2D) via i.p. injection for 14 days. On day 14, the mice were sacrificed and tumor weights were measured. Tumor weights were expressed as the means \pm SD of each group. Significance was determined via an ANOVA test vs. the vehicle group ( $\mathrm{P}<0.05)$. (C) A representative tumor image.

$R T$-PCR. Single-strand cDNA was synthesized from $2 \mu \mathrm{g}$ of total RNA, using AccuPower ${ }^{\circledR}$ RT premix (Bioneer, Korea). PCR amplification was subsequently performed in a reaction volume of $20 \mu \mathrm{l}$ containing $2 \mu \mathrm{l}$ of the appropriate cDNA, $1 \mu \mathrm{l}$ of each set of primers at a concentration of $10 \mathrm{pM}$ and Accupower PCR premix (Bioneer, Korea). The sequences of the primers used were: $H R K$, forward: 5'-TGCTCGGCAGGC GGAACTTGTAG-3' and reverse, 5'-GCTTCCCCAGTCCCA TTCTGTGTTT-3'; TNFRSF10B, forward: 5'-CTTGATTGT GGCTGTGTTTGTT-3' and reverse, 5'-GCCACCTTTATC TCATTGTCCA-3'; PYCARD, forward: 5'-CTCCTCAGTCGG CAGCCAAG-3' and reverse, 5'-GGAGTGTTGCTGGGAA GGAG-3'; TNFRSF8, forward: 5'-CTGTGTCCCCTACCCA ATCT-3' and reverse, 5'-CTTCTTTCCCTTCCTCTTCCA-3'. The RT-PCR products were electrophoresed on a $1.5 \%$ agarose gel (Mupid-2) and visualized via staining with ethidium bromide (GelDoc 1000, Bio-Rad), respectively.

Statistical analysis. The results were expressed as the means \pm SD. One-way ANOVA followed by Dunnett's t-test was employed for multiple comparisons using GraphPad Prism (GraphPad Software Inc., CA, USA). P<0.05 was considered to be statistically significant.

\section{Results}

Effect of KBH-A42 on human leukemia and bladder cancer cell lines. The effects of KBH-A42 on cell proliferation were assessed in 14 human cancer cell lines obtained from 9 different organs. Among them, the K562 human leukemia cells were the most sensitive to $\mathrm{KBH}-\mathrm{A} 42\left(\mathrm{GI}_{50}=1.41 \mu \mathrm{M}\right)$, whereas the UM-UC-3 bladder cancer cells were the least sensitive $\left(\mathrm{GI}_{50}>10 \mu \mathrm{M}\right)$ (Table I). $\mathrm{KBH}-\mathrm{A} 42$ inhibited the growth of K562 cells in a dose-dependent manner. However, the growth of UM-UC-3 cells was not affected at concentrations $<5 \mu \mathrm{M}$ of $\mathrm{KBH}-\mathrm{A} 42$ and was inhibited by only $11.3 \%$ at $10 \mu \mathrm{M}$ (Fig. 1A). Furthermore, to determine whether the in vitro effects of $\mathrm{KBH}-\mathrm{A} 42$ corresponded to the anti-tumor effects in vivo, we investigated whether KBH-A42 affected tumor growth in a human tumor xenograft model. As shown in Fig. 1B and C, KBH-A42 (100 mg/kg, QD) or ADR (2 mg/kg, Q2D) induced a 37 or $40 \%$ inhibition of the growth of K562 tumors, respectively. However, in the UM-UC-3 tumors, KBH-A42 and ADR inhibited growth by 21 or $67 \%$, respectively (Fig. $1 \mathrm{~B}$ and $\mathrm{C}$ ).

Induction of apoptosis and activation of caspases by $\mathrm{KBH}-\mathrm{A} 42$ in K562 and UM-UC-3 cells. To elucidate the mechanism underlying cell growth inhibition by $\mathrm{KBH}-\mathrm{A} 42$, the apoptosis profiles were analyzed via flow cytometry. To detect apoptotic induction by KBH-A42, the cells were stained with annexin V and PI. Whereas KBH-A42 induced apoptosis by as much as $36 \%$ at $10 \mu \mathrm{M}$ in the $\mathrm{K} 562$ cells, a $3.5 \%$ induction of apoptosis was noted in the UM-UC-3 cells even at $10 \mu \mathrm{M}$ (Fig. 2A and B). These results were consistent with the results shown in Fig. 2C, whereas KBH-A42 induced the activation of caspases 3/7 in K562 cells, but not in UM-UC-3 cells.

Analysis of gene expression changes caused by $\mathrm{KBH}-\mathrm{A} 42$ treatment. To gain insight into the mechanism by which $\mathrm{KBH}-\mathrm{A} 42$ inhibits cell growth, the mRNA expression profiles of $\mathrm{K} 562$ and UM-UC-3 cells were analyzed in the presence and absence of KBH-A42. Total RNAs were isolated from K562 and UM-UC-3 cells that were either left untreated or treated with $5 \mu \mathrm{M}$ KBH-A42 for $24 \mathrm{~h}$, labeled and hybridized to arrays (Agilent human 44K 4-plex chip), and at least 

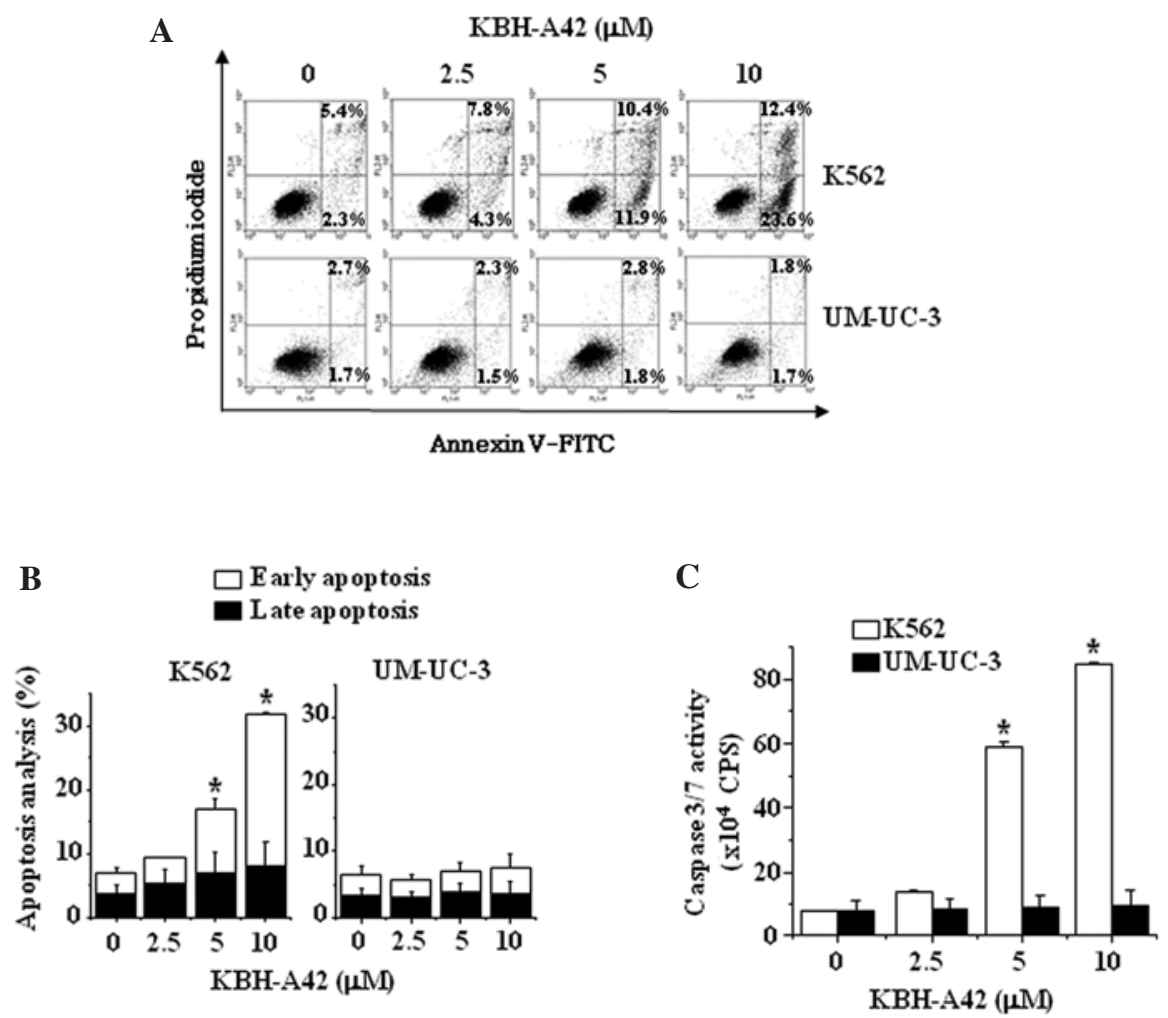

Figure 2. Apoptosis analysis by KBH-A42 in K562 and UM-UC-3 cells. Cells were treated for $24 \mathrm{~h}$ with KBH-A42 at the indicated concentrations. (A) Cells were stained with propidium iodide and a FITC-conjugated antibody directed against annexin V, and then analyzed by flow cytometry. (B) Results of flow cytometry are expressed as the means \pm SD of triplicate determinations. (C) The culture supernatants were collected, and the activity of caspases $3 / 7$ was determined. Significance was determined via an ANOVA test vs. DMSO-treated controls ( $\mathrm{P}<0.05)$.

Table II. Up- and down-regulated genes by KBH-A42 treatment in K562 and UM-UC-3 cells.

GeneBank number

Gene symbol

Fold change vs. control

K562

UM-UC-3

Up-regulated genes

NM_015193
NM_001165
NM_001252
NM_003805
NM_003806
NM_021960
NM_003842
NM_148968

$A R C$

7.71

2.49

BIRC3

12.25

2.23

CD70

5.06

1.13

CRADD

2.52

1.07

HRK

5.69

0.40

MCL1

3.43

0.91

TNFRSF $10 B$

5.30

2.65

TNFRSF 25

10.62

7.99

Down-regulated genes
NM_000633
NM_015367
NM_032587
BC010607
NM_013258
NM_032308
NM_133484
NM_001243
NM_005426

BCL2

0.35

1.39

BCL2L13

0.45

1.27

CARD6

0.18

0.79

IGFIR

0.47

1.26

PYCARD

0.20

1.42

RIP

0.44

1.60

TANK

0.41

1.38

TNFRSF8

0.14

0.76

TP53BP2

0.44 
a 2-fold difference was detected between the untreated and treated groups with regard to apoptosis-related genes. Among the 139 apoptosis-related genes assessed, $14(10.07 \%)$ and $17(12.23 \%)$ genes were up-regulated (ratio $>2.0$ ) in the K562 and UM-UC-3 cells, and 34 (24.46\%) and 42 (30.22\%) genes were down-regulated (ratio <0.5) in the K562 and UM-UC-3 cells. The most prominently up-regulated genes showing at least a two-fold difference between the K562 and UM-UC-3 cells treated with $\mathrm{KBH}-\mathrm{A} 42$ were: activityregulated cytoskeleton-associated protein $(A R C)$, baculoviral IAP repeat-containing 3 (BIRC3), CD70 molecule (CD70), CASP2 and RIPK1 domain-containing adaptor with death domain $(C R A D D)$, harakiri $(H R K)$, myeloid cell leukemia sequence 1 (MCL1), tumor necrosis factor receptor superfamily, member 10b (TNFRSF10B) and tumor necrosis factor receptor superfamily, member 25 (TNFRSF25) (Table II). The most profoundly down-regulated genes that also evidenced at least a 2-fold difference between K562 and UM-UC-3 cells were: B-cell CLL/lymphoma2 (BCL2), BCL2-like 13 (BCL2L13), caspase recruitment domain family, member 6 (CARD6), insulin-like growth factor 1 receptor (IGF1R), PYD and CARD domain containing protein gene (PYCARD), RPA interacting protein, transcript variant $2(R I P)$, TRAF family member-associated NF- $\mathrm{B}$ activator $(T A N K)$, tumor necrosis factor receptor superfamily, member $8(T N F R S F 8)$ and tumor protein p53 binding protein 2 (TP53BP2) (Table II).

Effect of $\mathrm{KBH}-\mathrm{A} 42$ on the expression of apoptosis-related genes. Since KBH-A42 induced apoptosis in K562 cells but not in UM-UC-3 cells, apoptosis-related genes that were altered by $\mathrm{KBH}-\mathrm{A} 42$ were selected from the cDNA microarray results and subjected to RT-PCR for confirmation. Two genes, HRK and TNFRSF10B, were up-regulated by $\mathrm{KBH}-\mathrm{A} 42$, as demonstrated by the results of the microarray analysis. The expression of $H R K$ mRNA was reduced in the UM-UC-3 cells, but increased slightly in the K562 cells. The expression of the TNFRSF $10 B$ mRNA was greatly increased as a result of $\mathrm{KBH}-\mathrm{A} 42$ treatment in the $\mathrm{K} 562$ cells, but was unaltered in the UM-UC-3 cells (Fig. 3). The mRNA expression of PYCARD and TNFRSF8, which was down-regulated by KBH-A42 in the cDNA microarray, was re-examined and confirmed via RT-PCR. PYCARD and TNFRSF10B mRNA showed a reduced expression as a result of $\mathrm{KBH}-\mathrm{A} 42$ treatment in the K562 cells, but was not detected in the UM-UC-3 cells (Fig. 3).

\section{Discussion}

In this study, we demonstrated that a novel $\delta$-lactam-based HDAC inhibitor, KBH-A42, inhibited the growth of 14 selected cancer cell lines. The findings described have shown that KBH-A42 significantly suppressed the growth of almost all of the tested cancer cell lines, but certain cell types were more susceptible to the compound than others. Whereas the leukemia cell lines were the most sensitive to KBH-A42, the glioma and prostate cancer cell lines were weakly sensitive, and the bladder cancer cell line was the least sensitive. Our findings demonstrate the cell type-specific growth inhibitory effect of KBH-A42. In a human tumor xenograft model using $\mathrm{Balb} / \mathrm{c}$ nude mice, $\mathrm{KBH}-\mathrm{A} 42$ has been shown to inhibit the
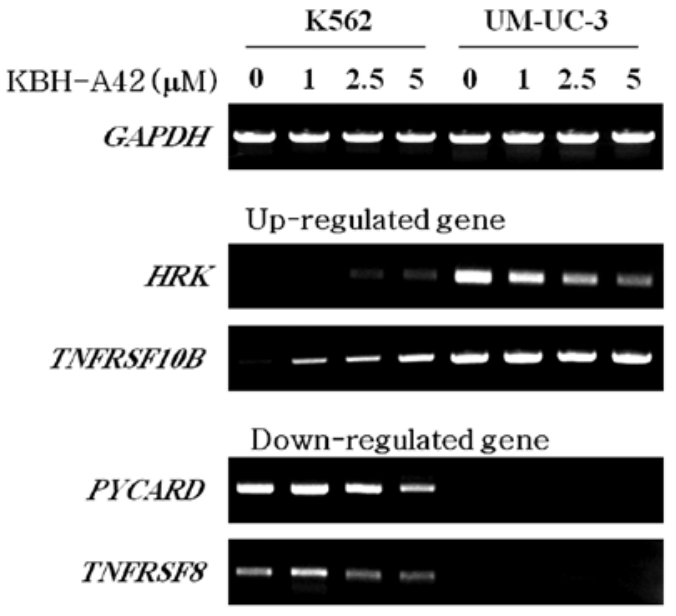

Figure 3. Confirmation of the up- and down-regulated genes in a cDNA microarray experiment by RT-PCR. Total RNA was isolated from K562 and UM-UC-3 cells either left untreated or treated with KBH-A42 (1, 2.5 and $5 \mu \mathrm{M}$ ) for $24 \mathrm{~h}$. RT-PCR was conducted and a total of $5 \mu \mathrm{l}$ of PCR products were run on $1.5 \%$ agarose gel. As an internal control, GAPDH was amplified.

growth of K562 tumors significantly, but only slightly inhibited the growth of UM-UC-3 tumors.

Since HDAC inhibitors have been reported to induce apoptosis in a variety of cancer cell lines (15-17), we evaluated the effect of KBH-A42 on the apoptosis of K562 and UM-UC-3 cells. The results of the apoptosis analysis demonstrate that the growth inhibition of K562 cells by KBH-A42 was mediated, at least in part, by the induction of apoptosis, but that of UM-UC-3 cells was not. The in vitro caspase assays, which measured the involvement of the major effectors of apoptosis, showed a significant difference between the activities of caspase $3 / 7$.

A cDNA microarray was conducted to screen for any changes in the expression of apoptosis-associated genes. The DNA microarray technique is a powerful method, allowing for the simultaneous analysis of the expression levels of multiple genes. In this study, gene expression was profiled using cDNA microarrays on KBH-A42-treated cells. A number of apoptosis-related genes were identified from the list of the genes modulated by KBH-A42 on the cDNA microarray assays. Among them, four genes that were up- or down-regulated by $\mathrm{KBH}-\mathrm{A} 42$ were selected and their expression patterns were confirmed via RT-PCR.

In the present study, 139 apoptosis-related genes whose expression was altered by $\mathrm{KBH}-\mathrm{A} 42$ treatment in $\mathrm{K} 562$ and UM-UC-3 cells were screened out. Eight genes that were up-regulated (ratio $>2.0$ ) and showed at least a 2-fold difference between the K562 and UM-UC-3 cells were apoptosis-associated genes. In mammalian cells, the prosurvival members (Bcl-2, Bcl-xL, Bcl-w, Mcl-1 and A1) oppose the pro-apoptotic members, which consist of the Bax group (Bax, Bak and Bok) and the BH3-only proteins (Bim, Bad, Bid, Bik, Bmf, Puma, Noxa and HRK). The BH3-only proteins monitor cellular well-being and, when activated by cytotoxic signals, engage pro-survival relatives by inserting the $\mathrm{BH} 3$ domain into a hydrophobic groove on their surfaces (18). The expression of HRK induced rapid cell death, which was repressed by $\mathrm{Bcl}-2$ and $\mathrm{Bcl}-\mathrm{xL}$. It was determined that 
the deletion of the $\mathrm{BH} 3$ region eliminated the ability of HRK to interact with Bcl-2 or Bcl-xL and eliminated or profoundly attenuated HRK killing activity (19). In this study, HRK was slightly up-regulated in K562 cells but significantly down-regulated in UM-UC-3 cells as a result of KBH-A42 treatment. In addition, Takimoto et al previously asserted that TNFRSF10B was a viable tumor suppressor candidate, and the overexpression of TNFRSF1OB induced apoptosis in tumor cells in a p53-dependent manner (20). The results of this study have shown that KBH-A42 up-regulated the expression of TNFRSF1OB in K562 cells in a concentration-dependent manner, but caused no such changes in UM-UC-3 cells.

In addition, nine genes were found to be down-regulated (ratio <0.5) and exhibited more than a 2-fold difference between the K562 and UM-UC-3 cells; these cells were also associated with apoptosis. Apoptosis-associated speck-like protein containing a $\mathrm{C}$-terminal caspase-recruitment domain (CARD) is a protein encoded by the PYCARD gene in humans. This gene encodes for an adaptor protein composed of a PYD and a CARD domain (21). PYCARD mediates the assembly of large signaling complexes in the inflammatory and apoptotic signaling pathways, via caspase activation. In K562 cells, PYCARD was reduced in a dose-dependent manner and was not detected in UM-UC-3 cells. TNFRSF8, which is involved in apoptosis, is a member of the TNF receptor superfamily, which is capable of inducing apoptosis by activating the caspase pathway (22). In K562 cells, TNFRSF 8 was reduced in a dose-dependent manner and was not detected in UM-UC-3 cells. Thus, KBH-A42 may inhibit cell growth by regulating the expression of apoptosis-related genes, and may have better efficacy in leukemia.

In conclusion, the results of this study have demonstrated that KBH-A42 inhibited the growth of cancer cells such as $\mathrm{K} 562$ cells under in vitro and in vivo conditions, and also that the growth inhibitory effects of KBH-A42 appear to be mediated by apoptosis. In the microarray assays, apoptosisrelated genes containing $A R C, B I R C 3, C D 70, C R A D D, H R K$, MCL1, TNFRSF10B and TNFRSF25 were up-regulated and BCL2, BCL2L13, CARD6, IGF1R, PYCARD, RIP, TANK, $T N F R S F 8$ and TP53BP2 were down-regulated. Among these genes, changes in the expression of HRK, TNFRSF10B, PYCARD and TNFRSF $8 \mathrm{mRNAs}$ was confirmed via RT-PCR. Collectively, our results have shown that KBHA42 possesses anti-cancer properties and has a strong potential as a therapeutic candidate with improved efficacy on leukemia under in vitro and in vivo conditions. However, different cells may be regulated differentially by $\mathrm{KBH}-\mathrm{A} 42$.

\section{Acknowledgements}

This study was supported by the Bio R\&D program through the Korea Science and Engineering Foundation funded by the Ministry of Education, Science and Technology (2008-0029594) and the KRIBB Research Initiative program.

\section{References}

1. Saito A, Yamashita T, Mariko Y, et al: A synthetic inhibitor of histone deacetylase, MS-27-275, with marked in vivo antitumor activity against human tumors. Proc Natl Acad Sci USA 96: 4592-4597, 1999.
2. Saunders NA, Popa C, Serewko MM, Jones SJ, Dicker AJ and Dahler AL: Histone deacetylase inhibitors: novel anticancer agents. Expert Opin Investig Drugs 8: 1611-1621, 1999.

3. Grunstein M: Histone acetylation in chromatin structure and transcription. Nature 389: 349-352, 1997.

4. Chambers AE, Banerjee S, Chaplin T, et al: Histone acetylationmediated regulation of genes in leukaemic cells. Eur J Cancer 39: 1165-1175, 2003.

5. Glaser KB, Staver MJ, Waring JF, Stender J, Ulrich RG and Davidsen SK: Gene expression profiling of multiple histone deacetylase (HDAC) inhibitors: defining a common gene set produced by HDAC inhibition in T24 and MDA carcinoma cell lines. Mol Cancer Ther 2: 151-163, 2003.

6. Mitsiades CS, Mitsiades NS, McMullan CJ, et al: Transcriptional signature of histone deacetylase inhibition in multiple myeloma: biological and clinical implications. Proc Natl Acad Sci USA 101: 540-545, 2004.

7. Peart MJ, Smyth GK, van Laar RK, et al: Identification and functional significance of genes regulated by structurally different histone deacetylase inhibitors. Proc Natl Acad Sci USA 102: 3697-3702, 2005.

8. Sasakawa Y, Naoe Y, Sogo N, et al: Marker genes to predict sensitivity to FK228, a histone deacetylase inhibitor. Biochem Pharmacol 69: 603-616, 2005.

9. Gray SG, Qian CN, Furge K, Guo X and Teh BT: Microarray profiling of the effects of histone deacetylase inhibitors on gene expression in cancer cell lines. Int J Oncol 24: 773-795, 2004

10. Butler LM, Agus DB, Scher HI, et al: Suberoylanilide hydroxamic acid, an inhibitor of histone deacetylase, suppresses the growth of prostate cancer cells in vitro and in vivo. Cancer Res 60: 5165-5170, 2000.

11. Richon VM, Sandhoff TW, Rifkind RA and Marks PA: Histone deacetylase inhibitor selectively induces p21WAF1 expression and gene-associated histone acetylation. Proc Natl Acad Sci USA 97: 10014-10019, 2000.

12. Kang MR, Kang JS, Han SB, et al: A novel delta-lactam-based histone deacetylase inhibitor, KBH-A42, induces cell cycle arrest and apoptosis in colon cancer cells. Biochem Pharmacol 78: 486-494, 2009

13. Kang MR, Lee K, Kang JS, et al: KBH-A42, a histone deacetylase inhibitor, inhibits the growth of doxorubicin-resistant leukemia cells expressing P-glycoprotein. Oncol Rep 23: 801-809, 2010.

14. Lee SD, Park SK, Lee ES, et al: A lipid-soluble red ginseng extract inhibits the growth of human lung tumor xenografts in nude mice. J Med Food 13: 1-5, 2010.

15. Louis M, Rosato RR, Brault L, et al: The histone deacetylase inhibitor sodium butyrate induces breast cancer cell apoptosis through diverse cytotoxic actions including glutathione depletion and oxidative stress. Int J Oncol 25: 1701-1711, 2004.

16. Doi S, Soda H, Oka M, et al: The histone deacetylase inhibitor FR901228 induces caspase-dependent apoptosis via the mitochondrial pathway in small cell lung cancer cells. Mol Cancer Ther 3: 1397-1402, 2004.

17. Roh MS, Kim CW, Park BS, et al: Mechanism of histone deacetylase inhibitor Trichostatin A induced apoptosis in human osteosarcoma cells. Apoptosis 9: 583-589, 2004.

18. Willis SN and Adams JM: Life in the balance: how BH3-only proteins induce apoptosis. Curr Opin Cell Biol 17: 617-625, 2005.

19. Inohara N, Ding L, Chen S and Nunez G: Harakiri, a novel regulator of cell death, encodes a protein that activates apoptosis and interacts selectively with survival-promoting proteins Bcl-2 and Bcl-X(L). EMBO J 16: 1686-1694, 1997.

20. Takimoto R and El-Deiry WS: Wild-type p53 transactivates the KILLER/DR5 gene through an intronic sequence-specific DNA-binding site. Oncogene 19: 1735-1743, 2000.

21. Stehlik C, Lee SH, Dorfleutner A, Stassinopoulos A, Sagara J and Reed JC: Apoptosis-associated speck-like protein containing a caspase recruitment domain is a regulator of procaspase-1 activation. J Immunol 171: 6154-6163, 2003.

22. Staber PB, Noehammer C, Durkop H, et al: mRNA expression patterns indicate $\mathrm{CD} 30$ mediated activation of different apoptosis pathways in anaplastic large cell lymphoma but not in Hodgkin's lymphoma. Leuk Res 30: 343-348, 2006. 